\title{
Ansatz from nonlinear optics applied to trapped Bose-Einstein condensates
}

\author{
Murat Keçeli, F. Ö. Ilday, and M. Ö. Oktel \\ Department of Physics, Bilkent University, 06800 Ankara, Turkey
}

(Received 30 November 2006; published 13 March 2007)

\begin{abstract}
A simple analytical ansatz, which has been used to describe the intensity profile of the similariton laser (a laser with self-similar propagation of ultrashort pulses), is used as a variational wave function to solve the Gross-Pitaevskii equation for a wide range of interaction parameters. The variational form interpolates between the noninteracting density profile and the strongly interacting Thomas-Fermi profile smoothly. The simple form of the ansatz is modified for both cylindrically symmetric and completely anisotropic harmonic traps. The resulting ground-state density profile and energy are in very good agreement with both the analytical solutions in the limiting cases of interaction and the numerical solutions in the intermediate regime.
\end{abstract}

DOI: 10.1103/PhysRevA.75.035601

PACS number(s): 03.75.Hh, 42.65.Tg

It is common for dynamical systems with weak coupling to show Gaussian behavior with respect to key parameters, whereas strongly coupled and highly nonlinear systems tend to exhibit power law dependences. A striking recent example of this is the ultrashort pulse formation in the similariton laser (a laser with self-similar propagation of ultrashort pulses) [1]. Another nonlinear system, which exhibits a parabolic density profile for strong interactions, is a trapped Bose-Einstein condensate (BEC). In Bose-Einstein condensation, the density of the condensate is analogous to the intensity of light in nonlinear optics (NLO) and the nonlinear governing equation for this system, which is called the Gross-Pitaevskii equation (GPE), is very similar to the equation for propagation of laser light in a nonlinear optical medium. Based on this similarity, it is natural to expect similar solutions for these vastly different systems. Solitonlike and self-similar solutions of the nonlinear Schrödinger equation (NLSE) are important both in BEC and NLO.

Solitonlike solutions arise when the nonlinearity is compensated by the dispersion and they are the only exact analytical solutions of these NLSE's, whereas self-similar solutions are asymptotic solutions that show up when the effects of initial conditions die out but the system is still far from the final state [2]. Although soliton-type solutions have been extensively studied in both the NLO and BEC communities, self-similar solutions are not as comprehensively investigated. In optics, these types of solutions are used more extensively from Raman scattering to pulse propagation in fibers, and it is shown that linearly chirped parabolic pulses are exact asymptotic solutions of the NLSE with gain [3]. Recently, we have demonstrated experimentally and numerically that self-similar propagation of ultrashort parabolic pulses (similaritons) are stable in a laser resonator [1]. More recently, we have developed a semianalytic theory of the similariton laser [4,5]. Instrumental in this step was the introduction of an ansatz to describe the intensity profile of this pulse, which can be "tuned" to any condition, ranging from weakly nonlinear (Gaussian pulses) to strongly nonlinear (parabolic pulses). Here, motivated by the mathematical similarity between the two systems, we apply the same ansatz to describe the density profile of a BEC in a quadratic trap. We show that this ansatz describes the system with excellent accuracy, in the whole range from the noninteracting limit to the strongly interacting limit.
Gross-Pitaevskii theory [6] gives a very successful description of the ground state and excitations of BEC's in dilute atomic gases. The success of this theory implies that the condensate can be described accurately with a single wave function and the interactions between the particles are through $s$-wave scatterings. The interaction of the particles are then represented by the interaction strength $g=\frac{4 \pi \hbar^{2} a}{m}$, where $a$ is the $s$-wave scattering length and $m$ is the mass of the trapped particles. The theory reduces to a single equation that describes the condensate wave function, known as the GPE, a type of nonlinear Schrödinger equation which arises in many areas of physics like NLO and hydrodynamic theory of fluids.

The GPE can be obtained by minimizing the ground-state energy functional of the condensate,

$$
E(\psi)=\int d \mathbf{r}\left(\frac{\hbar^{2}}{2 m}|\nabla \psi(\mathbf{r})|^{2}+V(\mathbf{r})|\psi(\mathbf{r})|^{2}+\frac{g}{2}|\psi(\mathbf{r})|^{4}\right),
$$

with respect to the wave function. The terms in the energy functional correspond to kinetic, trapping, and interaction energies, respectively. The trapping potential can generally be approximated with a harmonic potential for many of the experiments. The time-independent GPE follows as

$$
-\frac{\hbar^{2}}{2 m} \nabla^{2} \psi(\mathbf{r})+V(\mathbf{r}) \psi(\mathbf{r})+g|\psi(\mathbf{r})|^{2} \psi(\mathbf{r})=\mu \psi(\mathbf{r}),
$$

where $\mu$ is chemical potential introduced as the Lagrange multiplier for the normalization constraint $\int d \mathbf{r} n(\mathbf{r})=N$, where $n(\mathbf{r})=|\psi(\mathbf{r})|^{2}$ is the density of the condensate.

The nonlinearity of the GPE is due to an interaction between particles and its effect becomes more pronounced as the number of particles in the condensate increases which is the case for current experiments where more than $10^{7}$ particles form the BEC. Since very few exact solutions of the NLSE, such as solitons, are known, many numerical algorithms [7-11] and variational methods [12-17] are developed to find ground-state solutions. Although variational methods give only an upper bound to the ground-state energy, they require less calculation and can give accurate results if a suitable trial function is chosen. Another advantage of the 
variational principle is that it gives the functional form of the wave function which can be used to obtain other properties of the condensate.

Therefore, many trial functions are proposed to obtain a lower bound for the ground-state energy. Trial functions are generally chosen by adding parameters to a known approximate analytical solution. The trivial approximate solutions are obtained by looking at the limiting cases of the GPE where the nonlinearity is negligibly small or very high. Assuming an ideal Bose gas, Eq. (2) reduces to the Schrödinger equation where the chemical potential corresponds to the energy eigenvalue, by neglecting the nonlinear term. With a harmonic trap the problem turns into a harmonic oscillator problem where the solution is a Gaussian. For the opposite case where nonlinearity is dominant, the kinetic energy term can be neglected in the GPE, and then the equation can easily be solved for the density, $n(\mathbf{r})=\frac{\mu-V(\mathbf{r})}{2 g}$, when the righthand side of the equation is positive. This approximation is known as the Thomas-Fermi approximation (TFA), and it shows that as the interaction increases, the density profile changes from a Gaussian to a parabola for a harmonic trap. The TFA can be improved by adding the kinetic energy term obtained with the resulting wave function with a suitable cutoff $[18]$ as

$$
\frac{E_{k i n}}{N}=(15 \beta)^{-2 / 5}\left(\frac{1}{2} \ln (480 \beta)-\frac{5}{4}\right) .
$$

With this insight many trial functions are proposed for isotropic traps and anisotropic traps with cylindrical symmetry to describe the intermediate regime where neither the TFA and nor the ideal gas approximation is valid. Here, we make use of a simple analytic function which has already been used in NLO where a similar behavior-Gaussian to parabolic - for the intensity profile of a similariton laser.

The so-called similariton pulse in optics has a nearly parabolic intensity profile to reduce the effect of Kerr nonlinearity. However, if the nonlinearity of the system is reduced, the pulse assumes the well-known Gaussian shape of dispersionmanaged solitons. Therefore, the ansatz proposed in [4] to describe these pulses has an adjustable profile between a Gaussian and an inverted parabola:

$$
S_{n}(x)=\exp \left(-\sum_{k=1}^{n} \frac{x^{2 k}}{k}\right) .
$$

This function becomes a Gaussian when $n=1$,

$$
S_{n=1}(x)=\exp \left(-x^{2}\right),
$$

and turns into an inverted parabola when $n$ goes to $\infty$ since the summation in the exponent converges to $\ln \left(1-x^{2}\right)$ for $|x|<1$,

$$
S_{n \rightarrow \infty}=\exp \left(-\sum_{k=1}^{\infty} \frac{x^{2 k}}{k}\right)=\exp \left[\ln \left(1-x^{2}\right)\right]=1-x^{2} .
$$

Moreover, the expansion converges so quickly that adding about ten terms is enough to get a parabolic profile with smooth ends. Besides, this function is easily integrable which makes it a good candidate for variational calculations.
TABLE I. The values of the wave function at the center, the root-mean-square sizes $r_{r m s}$, and chemical potentials are tabulated in units of $\sqrt{N / a_{w}^{3}}, a_{w}$, and $\hbar \omega$, respectively. For comparison numerical results of Ref. [11] are given in parentheses.

\begin{tabular}{ccccccc}
\hline \hline$\beta$ & \multicolumn{2}{c}{$\psi(0)$} & \multicolumn{2}{c}{$r_{r m s}$} & \multicolumn{2}{c}{$\mu$} \\
\hline 0 & 0.4238 & $(0.4238)$ & 1.2248 & $(1.2248)$ & 1.5000 & $(1.5000)$ \\
0.2496 & 0.3969 & $(0.3843)$ & 1.2794 & $(1.2785)$ & 1.6805 & $(1.6774)$ \\
0.9986 & 0.3475 & $(0.3180)$ & 1.3981 & $(1.3921)$ & 2.0885 & $(2.0650)$ \\
2.4964 & 0.2515 & $(0.2581)$ & 1.5355 & $(1.5356)$ & 2.5803 & $(2.5861)$ \\
9.9857 & 0.1739 & $(0.1738)$ & 1.8822 & $(1.8821)$ & 4.0089 & $(4.0141)$ \\
49.926 & 0.1097 & $(0.1066)$ & 2.5071 & $(2.5057)$ & 7.2576 & $(7.2484)$ \\
249.64 & 0.0665 & $(0.0655)$ & 3.4152 & $(3.4145)$ & 13.559 & $(13.553)$ \\
2496.4 & 0.0330 & $(0.0328)$ & 5.3855 & $(5.3852)$ & 33.812 & $(33.810)$ \\
\hline \hline
\end{tabular}

Motivated by these properties, we use the similariton ansatz, Eq. (4), as our trial wave function to minimize the energy functional given in Eq. (1). To simplify the calculations, we non-dimensionalize the Gross-Pitaevskii functional by scaling length, energy, and wave function with oscillator length $a_{\omega}=\sqrt{\frac{\hbar}{m \omega}}, \hbar \omega$, and $\sqrt{N / a_{\omega}^{3}}$, respectively (see Table I). We first analyze the solution for a spherical harmonic trap $V(r)=\frac{1}{2} m \omega^{2} r^{2}$ and introduce the parameter $\beta \equiv N a / a_{\omega}$ which is a measure of the strength of the interaction. With this rescaling the energy functional becomes

$$
\frac{E(\psi)}{N}=2 \pi \int_{0}^{\infty} d^{3} r\left[|\nabla \psi(r)|^{2}+V(r)|\psi(r)|^{2}+2 \pi \beta|\psi(r)|^{4}\right] .
$$

Ideally $\beta$ can take any value between $-\infty$ to $\infty$ since all the parameters are experimentally tunable. However, a negative scattering length, which means an attractive interaction,

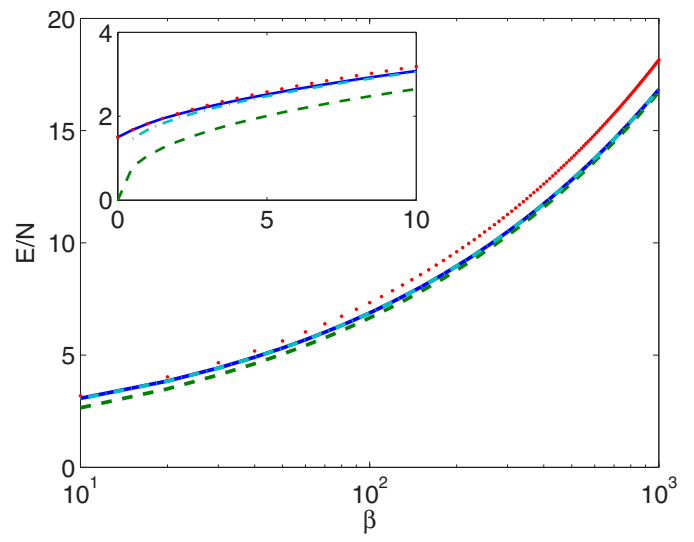

FIG. 1. (Color online) Ground-state energy with respect to the interaction parameter $\beta$ obtained with the variational function (black solid line). The resulting energy of a Gaussian variational function is given by dotted (red) line and energy obtained with Thomas-Fermi solution is given with dashed line (green). Improved Thomas-Fermi solution [18] is given by the dotted line (blue). Energy per particle is given in units of $\hbar \omega$. The inset is given for small $\beta$ values. 


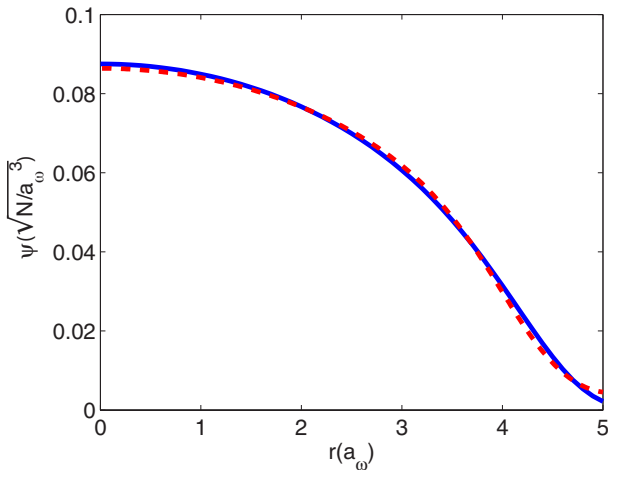

FIG. 2. (Color online) Wave function calculated with the steepest descent method [9] is shown as a bold line (blue) whereas the similariton ansatz solution is given by the dashed line (red) for $\beta=100$.

causes collapse of the condensate when the particle number is high. In this regime, our results agree with [12]. In the present work, we concentrate on repulsive interaction. With proper normalization the trial wave function has the form

$$
\psi(r)=\sqrt{\frac{1}{4 \pi d^{3} I}} \exp \left(\sum_{k=1}^{n} \frac{(r / d)^{2 k}}{2 k}\right),
$$

where $d$ and $n$ are our variational parameters with $I=\int_{0}^{\infty} d r r^{2} S_{n}(r)$ which is an integral that can be calculated analytically for $n=1,2$ and numerically for $n>2$. Here the parameter $d$ is responsible for the width of the condensate, which increases as the interaction increases, and $n$ takes care of flattening of the central density. We minimize the energy with respect to $d$ for different $n$ values and chose the $n$ that gives the minimum energy. For $d$, we obtain a fifth-order polynomial equation where only one of the roots is physically meaningful.

We compare our results with the analytical approximations as follows. For small $\beta$ values, our trial function reduces to a Gaussian and gives an exact result for $\beta=0$, and for large $\beta$ our results agree well with the improved TFA
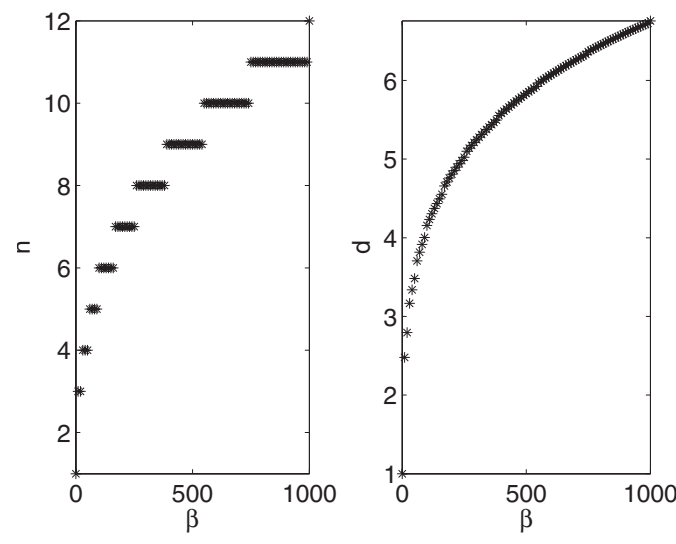

FIG. 3. Change of variational parameters with interaction parameter $\beta$ is given. Left plot shows the number of terms in the summation and the right one shows the change of width of the similariton ansatz in units of oscillator length $a_{\omega}$.

results as shown in Fig. 1. We also compare the resulting wave function with the numerical solutions obtained by the steepest descent method for different $\beta$ values in Fig. 2. We also tabulate our results and include the results of a recent numerical analysis which minimizes the energy functional directly by the finite-element method. Here it should be noted that tabulated kinetic, trap, and interaction energies satisfy the virial theorem $2 E_{k i n}+2 E_{t r}-3 E_{\text {int }}=0$, to our numerical accuracy. It is also remarkable that even for large $\beta$ adding ten terms is enough to find a good approximation for the wave function (see Fig. 3) which shows the simplicity of the calculations.

Using similar trial functions, we can also solve the GPE for a cylindrical trap and a fully anisotropic trap. For the cylindrically symmetric trap, trial function takes the form,

$$
\psi(\rho, z)=C \exp \left(-\sum_{k=1}^{n_{\rho}} \frac{\left(\rho / d_{\rho}\right)^{2 k}}{2 k}\right) \exp \left(-\sum_{k=1}^{n_{z}} \frac{\left(z / d_{z}\right)^{2 k}}{2 k}\right),
$$

where $C=\sqrt{\frac{N}{2 \pi d_{\rho}^{2} d_{z} I_{\rho} I_{z}}}, I_{\rho}=\int_{0}^{\infty} \rho d \rho S_{n}(\rho)$, and $I_{z}=\int_{-\infty}^{\infty} d z S_{n}(z)$. We have four variational parameters, but calculations are similar

TABLE II. Results of our calculation for a cylindrically harmonic trap with $\lambda=\sqrt{8}$. Energy and length units are $N \hbar \omega$ and $a_{\omega}$. The results of the numerical calculation in Ref. [9] are given in parentheses for comparison except for the last row. For $\beta=2165$ the Thomas-Fermi result

\begin{tabular}{|c|c|c|c|c|c|c|c|c|c|c|c|c|}
\hline \multirow{2}{*}{$\begin{array}{c}\beta \\
0.0000\end{array}$} & \multicolumn{2}{|c|}{$x_{r m s}$} & \multicolumn{2}{|c|}{$z_{r m s}$} & \multicolumn{2}{|c|}{$E_{k i n}$} & \multicolumn{2}{|c|}{$E_{t r}$} & \multicolumn{2}{|c|}{$E_{i n t}$} & \multicolumn{2}{|c|}{$\mu$} \\
\hline & 0.7071 & $(0.707)$ & 0.4204 & $(0.42)$ & 1.2071 & (1.207) & 1.2071 & (1.207) & 0.0000 & $(0.000)$ & 2.4142 & $(2.414)$ \\
\hline 0.4330 & 0.7901 & $(0.79)$ & 0.4374 & $(0.44)$ & 1.0539 & $(1.06)$ & 1.3894 & (1.39) & 0.2237 & $(0.21)$ & 2.8907 & $(2.88)$ \\
\hline 0.8660 & 0.8500 & $(0.85)$ & 0.4472 & $(0.45)$ & 0.9976 & $(0.98)$ & 1.5225 & $(1.52)$ & 0.3500 & $(0.36)$ & 3.2200 & $(3.21)$ \\
\hline 2.1650 & 0.9657 & $(0.96)$ & 0.4707 & $(0.47)$ & 0.8528 & $(0.86)$ & 1.8188 & $(1.81)$ & 0.6440 & $(0.63)$ & 3.9596 & (3.94) \\
\hline 4.3300 & 1.0892 & $(1.08)$ & 0.4966 & $(0.50)$ & 0.7337 & $(0.76)$ & 2.1730 & $(2.15)$ & 0.9595 & $(0.96)$ & 4.8258 & $(4.77)$ \\
\hline 8.6600 & 1.2319 & $(1.23)$ & 0.5332 & $(0.53)$ & 0.6709 & $(0.66)$ & 2.6549 & $(2.64)$ & 1.3227 & $(1.32)$ & 5.9712 & $(5.93)$ \\
\hline 21.650 & 1.4798 & $(1.47)$ & 0.5930 & $(0.59)$ & 0.5314 & $(0.54)$ & 3.5963 & $(3.57)$ & 2.0432 & $(2.02)$ & 8.2142 & (8.14) \\
\hline 43.300 & 1.7038 & (1.69) & 0.6536 & $(0.65)$ & 0.4351 & $(0.45)$ & 4.6121 & $(4.57)$ & 2.7847 & $(2.74)$ & 10.616 & $(10.5)$ \\
\hline 64.950 & 1.8447 & $(1.84)$ & 0.6989 & $(0.70)$ & 0.4128 & $(0.41)$ & 5.3569 & $(5.31)$ & 3.2960 & $(3.26)$ & 12.361 & $(12.2)$ \\
\hline 86.600 & 1.9562 & (1.94) & 0.7319 & $(0.73)$ & 0.3789 & $(0.38)$ & 5.9693 & $(5.91)$ & 3.7270 & $(3.68)$ & 13.802 & (13.7) \\
\hline 2165 & 3.73 & 367 & 1.3 & 297 & 0.1 & 459 & 21. & 035 & 13. & 926 & 49.033 & $(48.329)$ \\
\hline
\end{tabular}
for the chemical potential is given in parentheses. 
TABLE III. The chemical potentials per particle in units of $\hbar \omega$ are calculated using an ansatz modified for a completely anisotropic trap with $\lambda=\sqrt{2}$ and $g \gamma=2$. The values of the interaction parameter $\beta$ are obtained from Ref. [19], and the values in parentheses correspond to the numerical solution given in [10]. The variational parameters $n_{x, y, z}$ and $d_{x, y, z}$ are given.

\begin{tabular}{cccccc}
\hline \hline$\beta$ & $n_{x}, d_{x}$ & $n_{y}, d_{y}$ & $n_{z}, d_{z}$ & \multicolumn{2}{c}{$\mu$} \\
\hline 0 & $1,1.000$ & $1,0.840$ & $1,0.707$ & 2.207 & $(2.207)$ \\
1.787 & $2,1.753$ & $2,1.368$ & $1,0.817$ & 3.604 & $(3.572)$ \\
3.575 & $2,1.956$ & $2,1.489$ & $2,1.160$ & 4.385 & $(4.345)$ \\
7.151 & $3,2.433$ & $2,1.654$ & $2,1.258$ & 5.492 & $(5.425)$ \\
14.302 & $3,2.780$ & $3,2.035$ & $2,1.384$ & 7.010 & $(6.904)$ \\
28.605 & $4,3.310$ & $3,2.30$ & $3,1.687$ & 9.049 & $(8.900)$ \\
57.211 & $5,3.880$ & $4,2.725$ & $3,1.896$ & 11.78 & $(11.57)$ \\
\hline \hline
\end{tabular}

to the isotropic case. We compare our results with the numerical results of Dalfovo and Stringari [9] in Table II. Cylindrically symmetric traps are the most common traps in BEC setups, and the aspect ratio obtained from $\frac{x_{r m s}}{z_{r m s}}$ is very important to identify the BEC phase in these experiments. It is shown in $[9,12]$ that for the noninteracting case this ratio is equal to $\sqrt{\lambda}$ and goes to $\lambda$ in the Thomas-Fermi limit. This result is clearly seen from the values in Table II where $\lambda=\sqrt{8}$, and it is also evident that convergence of the TFA is very slow.

There are also experiments with fully anisotropic traps [19], and for this case the trial function can also be modified similarly with six variation parameters. The results are given in Table III, where agreement with the results in [10] is apparent.
In summary, an ansatz is introduced to investigate the ground-state properties of a BEC at zero temperature for quadratic traps with arbitrary anisotropy. The ground-state energy and wave function are found to be very accurate anywhere from the noninteracting case to the highly repulsive one, as compared with numerical studies. The form of the trial function changes from a Gaussian to a parabola smoothly, and it successfully describes the intermediate regime of moderate interaction. Important quantities like aspect ratio, chemical potential, and root-mean-square size of the clouds are calculated and compared to numerical studies [9-11]. With a slight modification the suggested form of the wave function can be applied to vortex states of the condensate. The time-dependent GPE can also be solved using a similar form to study the growth dynamics.

We have shown previously that a nonlinear system in optics-namely, a high-energy femtosecond laser oscillator-exists stably between two extreme limits, corresponding to Gaussian pulse profiles for weak nonlinearity and parabolic profiles for strong nonlinearity. This indeed appears to be a common behavior observed in many systems, including the trapped BEC analyzed in this paper. We have a simple analytical function, which has the crucial property of interpolating any state between these extremes. In this paper, we have shown that the variational approach with the same ansatz yields excellent results for the BEC system in a quadratic trap. We believe that our approach can be generally applicable to other nonlinear systems in disparate fields.

F.Ö.I. is supported by a TUBA-GEBIP grant and TUBITAK Grant No. 104T017. M.Ö.O. is supported by a TUBA-GEBIP grant and TUBITAK-KARIYER Grant No. $104 \mathrm{~T} 165$.
[1] F. Ö. Ilday, J. R. Buckley, W. G. Clark, and F. W. Wise, Phys. Rev. Lett. 92, 213902 (2004).

[2] G. I. Barenblatt, Scaling, Self-similarity, and Intermediate Asymptotics (Cambridge University Press, Cambridge, England, 1996).

[3] M. E. Fermann, V. I. Kruglov, B. C. Thomsen, J. M. Dudley, and J. D. Harvey, Phys. Rev. Lett. 84, 6010 (2000).

[4] C. Jirauschek, F. Ö. Ilday, and F. X. Kärtner (unpublished).

[5] C. Jirauschek and F. Ö. Ilday (unpublished).

[6] L. P. Pitaevskii, Sov. Phys. JETP 13, 451 (1961); E. P. Gross, Nuovo Cimento 20, 454 (1961); J. Math. Phys. 4, 195 (1963).

[7] M. Edwards and K. Burnett, Phys. Rev. A 51, 1382 (1995).

[8] P. A. Ruprecht, M. J. Holland, K. Burnett, and M. Edwards, Phys. Rev. A 51, 4704 (1995).

[9] F. Dalfovo and S. Stringari, Phys. Rev. A 53, 2477 (1996).

[10] B. I. Schneider and D. L. Feder, Phys. Rev. A 59, 2232 (1999).

[11] W. Bao and W. Tang, J. Comput. Phys. 187, 230 (2003).
[12] G. Baym and C. J. Pethick, Phys. Rev. Lett. 76, 6 (1996).

[13] K. N. Ilinski and A. Moroz, J. Res. Natl. Inst. Stand. Technol. 101, 567 (1996).

[14] V. M. Perez-Garcia, H. Michinel, J. I. Cirac, M. Lewenstein, and P. Zoller, Phys. Rev. Lett. 77, 5320 (1996); Phys. Rev. A 56, 1424 (1997).

[15] A. L. Fetter, J. Low Temp. Phys. 106, 643 (1997).

[16] J. L. Bohn, B. D. Esry, and C. H. Greene, Phys. Rev. A 58, 584 (1998).

[17] M. P. Singh and A. L. Satheesha, Eur. Phys. J. D 7, 391 (1999).

[18] E. Lundh, C. J. Pethick, and H. Smith, Phys. Rev. A 55, 2126 (1996).

[19] M. Kozuma, L. Deng, E. W. Hagley, J. Wen, R. Lutwak, K. Helmerson, S. L. Rolston, and W. D. Phillips, Phys. Rev. Lett. 82, 871 (1999). 